\title{
Defect Generation in Bipolar Devices by Ionizing Radiation
}

\author{
${ }^{1}$ Fuei Pien Chee, ${ }^{2}$ Haider F. Abdul Amir, ${ }^{3}$ Saafie Salleh \\ ${ }^{1,2,3}$ School of Sceince and Technology, University of Malaysia Sabah, Malaysia
}

\begin{abstract}
This paper reviews typical effects occurring in bipolar junction transistors (BJTs) due to gamma ( $\gamma$ ) rays irradiation. The detrimental consequences of this interaction can be categorized into two: the transfer of energy to electrons due to ionization and electronic excitations; and also the transfer of energy to atomic nuclei. The radiation damage induced by this interaction was studied using in situ method by comparing the currentvoltage characteristics of the devices under test (DUTs) at different biased collecting current and operating modes. The high energy from gamma radiation is found to induce both temporarily and permanent damage in the DUTs depending on the current drive and total dose absorbed. The most significant radiation damage in the $B J T$ is the creation of electron-hole pairs which increases the probability of recombination at the base region of BJTs. The DUTs are found to exhibit minor annealing effect at post-irradiation and the results also show that devices operating in higher bias current are more capable of withstanding the effect by gamma radiation.
\end{abstract}

Keywords: bipolar junction transistor (BJTs), gamma $(\gamma)$ rays, in situ, recombination, ionizing radiation

\section{Introduction}

Space technology has become an important part of everyday life. Technologies such as weather forecasting, remote sensing, global navigation positioning, satellite television and data broadcasting, telecommunications systems, as well as our surveillance and army based national security technologies are critically relied on space infrastructures. However, operating environment of these equipment and devices are far from stable and not without interference, especially in outer space [1,2]. The radiation environment that these systems might encounter either at exo-atmospheric or endo-atmoshperic can be generally divided into five classes which are the space environment, high-energy physic experiments, nuclear environment, natural environments as well as process induced radiation. Each of these radiation environments possesses its own spectrum of particles and energy distribution.

The first study using semiconductors as expedient structures to explore the radiation effects originated in the late 1940s. This research area is then continued through the following decades. Various types of radiation such as alpha $(\alpha)$ particles, gamma rays, neutrons and electrons had been used to observe the production of lattice-displacement defects in bulk crystalline semiconductor materials [3, 4]. The measurement techniques, for example, optical absorption, electronic transport, and electronic spin resonance (ESR) had been used to study the lattice defects on material properties [5]. This made the nuclear cross sections and thresholds for atomic displacement reactions and also the energy corresponding to the various effects could be determined. The annealing effect by radiation induced damage, however, was normally investigated over a range of temperatures. The secondary effects on lifetime degradation of the semiconductor devices which include the radiation induced degradation and carrier removal were also being studied.

The experimental studies were also emphasized to understand the effects of radiation induced Single Event Effects (SEEs) which occur due to a single, energetic particle and can be classified into three effects:

I. SEU or soft error is defined as radiation-induced errors in semiconductor devices caused when charged particles (usually from radiation belts or from cosmic rays) lose energy by ionizing the medium through which they pass, leaving behind a wake of electron-hole pairs. An SEU may occur in analog, digital, or optical components, or may have effects in surrounding interface circuitry [6]. SEUs typically appear as transient pulses in logic or support circuitry, or as bit flips in memory cells or registers. SEU may cause data corruption and alter program depending on the location of the upset [7]. A reset or rewriting of the device results in normal device behavior thereafter. The SEU itself is not considered permanently damaging to the transistor's or circuits' functionality unlike the case of single event latch-up (SEL), single event gate rupture (SEGR), or single event burnout (SEB).

II. SEL is a potentially destructive condition involving parasitic circuit elements forming silicon controlled rectifier (SCR). This may cause loss of device functionality due to a single-event induced current state. SELs are hard errors, and are potentially destructive [8]. The SEL results in a high operating current, above device specifications. The latched condition can destroy the device, drag down the bus voltage, or damage the power supply.

III. SEB is a condition that can cause device destruction due to a high current state in a power transistor. This event may occur when the passage of a single heavy ion forward biases the thin body region under the source of the device [9]. If the drain-to-source voltage of the device exceeds the local breakdown 
voltage of the parasitic bipolar, the device can burn out due to large currents and high local power dissipation. SEB's effects also include gate rupture, frozen bits, and noise. SEB susceptibility has been shown to decrease with increasing temperature.

Most of the previous work in the radiation damage in bipolar technologies was concerned with the displacement damage from heavy and light charged particles and also the total dose effects. However, the failure rate of the bipolar devices in the radiation prone environment is still a major concern in design and conceptualization of bipolar technology as many bipolar linear circuit normal functioning is damaged at high dose rate of 50-300 rad/s. This is known as enhanced low dose rate sensitivity (ELDRS) effect and has been actively investigated since the last decade [4].

The current approach to safeguard proper function is by shielding of such critical system or changing the material of electronic components. As the system is becoming increasingly complicated while the size of the chips is getting smaller, the shielding of all the systems and the material approach is becoming harder and less economical to be implemented into the design or building of such system. Since most of the research and studies are emphasizing on shielding and material study on the bipolar technology, we extended our studies to the damage extents of gamma radiation at different operating conditions and parameters. All the monitoring jobs are carried out in situ and this is different from some of the conventional measurement methods that the changes in output parameter of the DUTs are analyzed after irradiated by the source $[10,11]$. This conventional method provides less accuracy results because changes could only be monitored for not in-flux test.

\section{Gamma Irradiation}

Gamma irradiation, utilizing ${ }^{60} \mathrm{Co}$ was used to simulate the effect of ionizing radiation on BJTs. Ionizing radiation effect was studied as this radiation is so far considered as one of the primary concerns of the electronic components system failure in space or in a radiation environment [12]. Exposure on the DUTs was performed using the ${ }^{60} \mathrm{Co}$ from RAYMINTEX Plant, Malaysian Nuclear Agency. The outlook sketch of ${ }^{60} \mathrm{Co}$ gamma sources facility is as shown in Fig. 1.

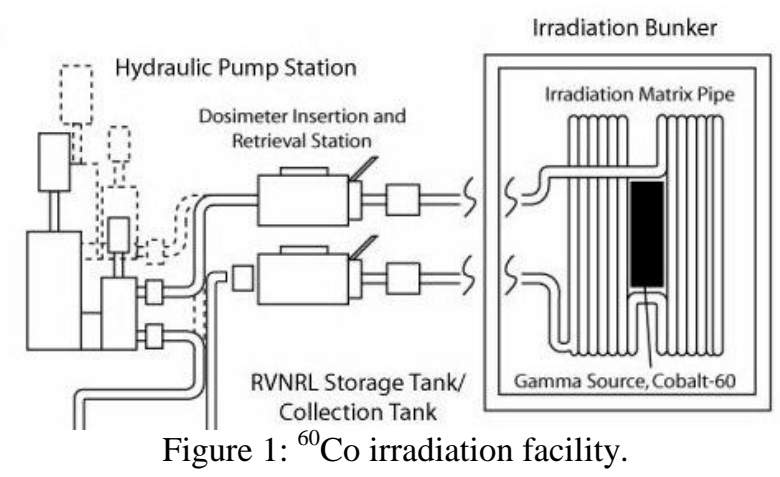

Thermoluminescence Dosimetry (TLD) consisting of lithium fluoride dosimeters was used for determining the absorbed dose. Absorbed dose is defined as the energy absorbed locally per unit mass due to the exposure to ionizing radiation. Several TLD tests were performed before determining the irradiation point from the table mode gamma irradiation. The special configuration and positioning of the coordinated table mode irradiation controlled the distance between the DUT and ${ }^{60}$ Co sources. This allowed equal exposure of radiation at a pre-determined constant dose rate. The dose rate at this selected point could be determined based on (1).

$$
\text { DoseRate }(\mathrm{rad} / \mathrm{s})=\frac{\text { DoseAbsorbed }(\mathrm{rad})}{\text { ExposureTime }(\mathrm{s})}
$$

A fixed point for locating the DUT at this ${ }^{60} \mathrm{Co}$ gamma field is crucial. This is as field uniformity and intensity can be affected by changes in the location of the device with respect to the radiation source and the presence of radiation absorption and scattering materials [13]. Since the dose rate at that particular point was known, the exposure time to irradiate the DUT up to a total absorbed dose of $60 \mathrm{krad}$ can be calculated. The dose rate of the activity shows a variation at different time due to the half-life of ${ }^{60} \mathrm{Co}$. However, this variation was not significant for tests which were conducted at a same period. The dose rate to reach a total dose of 60 $\mathrm{krad}(\mathrm{Si})$ in this exposure process was $5.0667 \mathrm{rad} / \mathrm{s}$ and the duration taken was 3 hours 17 minutes 22 seconds. After irradiation, a post-irradiation not in-flux test was run for 8 hours.

The DUTs were irradiated up to a total dose $60 \mathrm{krad}$ with reference to the NASA report from Poivey [14]. In this research paper, a more accurate estimation of the radiation level using 3D Monte Carlo code is utilized to calculate the total dose level received by the part at geostationary orbit. According to the analysis, the 
highest 15 years dose level simulated from Monte Carlo code is $24.0 \mathrm{krad}$ whereas those simulated from sectorial analysis- shell sphere/norm model and solid sphere/slant model is $28.5 \mathrm{krad}$ and $18.6 \mathrm{krad}$ respectively. From this data, the total dose of $60 \mathrm{krad}$ is sufficient to cater to most stringent condition in the proposed space environment.

\section{Radiation Exposure Procedures}

The radiation testing on the electronic devices consisted of multi-parameter test with different exposure levels. Ambient temperature throughout the test was $25 \pm 3^{\circ} \mathrm{C}$. Before the irradiation process, a control test of 72 hours known as the pre-irradiation testing was performed on the DUTs. Only devices which were within the specific working range and the testing results which reveal that they were within the standard deviation of 2 sigma were submitted for radiation testing. The radiation procedure was referred to the Military Standard (MILSTD) 883G Method 1019.7, Ionizing Radiation (Total Dose) Test Procedure [13].

The parameters $I_{C}$ and $I_{B}$ of the BJTs tested were monitored at input bias $I_{C}$ of $5 \mathrm{~mA}, 10 \mathrm{~mA}, 15 \mathrm{~mA}$ and $20 \mathrm{~mA}$. BJT NPN 2N3904 was used in this study. The schematic of experimental setup for the BJT is as shown in Fig. 2.

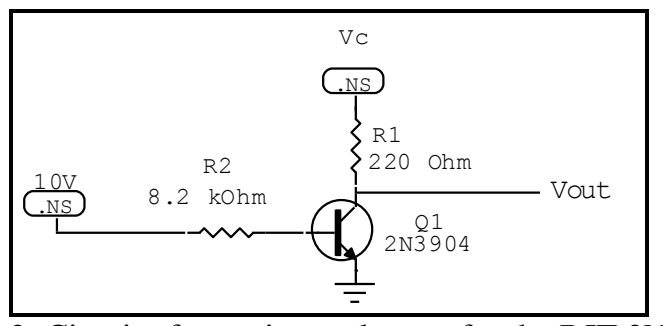

Figure 2: Circuit of experimental setup for the BJT 2N3904.

Every single test at different bias condition and irradiation mode was repeated 10 times using brand new devices. Two types of tests were performed in this experiment:

\section{a. In-flux test}

This in-flux test can be divided into two modes which are the ON Mode radiation and the OFF Mode radiation. In ON Mode radiation, measurements on the DUTs were done during radiation exposure that the DUTs were in operating condition. Therefore, all the changes in the parameters could be recorded and monitored directly. In OFF Mode radiation, however, the DUTs were irradiated in non-operating condition and the changes in parameters were monitored after irradiation. These tests were to compare the different effects of ionizing radiation on their operating mode.

\section{b. $\quad$ Not in-flux test}

Measurement made on DUTs after the irradiation which is known as post-irradiation. This is crucial for low dose rate or some applications in which the DUT may reveal time-dependent effects.

The input voltage of the particular device could be changed from a distance of approximately $15 \mathrm{~m}$ in a control room during irradiation and the effect could be observed directly using in-situ method. The schematic drawing of the test setup for in-situ testing is shown in Fig. 3. The information and status of the device under test (DUT) will be transmitted through the developed driver circuit based on an ADC circuit into the PC. The temperature dependence of the charge deposition in the sampling device would be monitored with an electronic thermometer using LM35 precision centigrade temperature sensor. All the data was transmitted directly to the computer. 


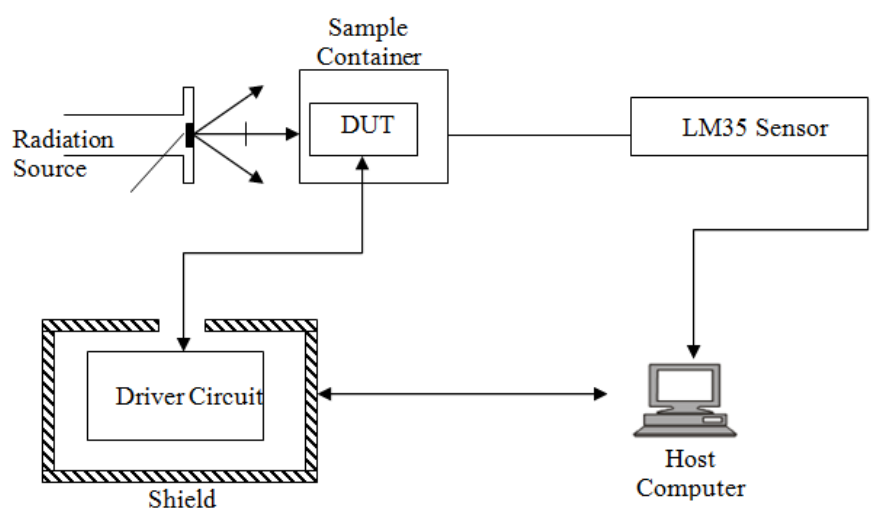

Figure 3: Experimental arrangement for observation of changes in the DUTs during irradiation.

\section{Radiation Effect In Common-Emitter Transfer Characteristics Of BJT}

The common-emitter current gain, $h_{f e}$ of BJT can be related to $I_{C}$ and $I_{B}$ through the (2)

$$
\frac{I_{C}}{I_{B}}=h_{f e}
$$

Fig. 4 and Fig. 5 show the $4\left(\frac{1}{h_{f e}}\right)$ of 2 N3904 for ON mode radiation during the exposure and also at postirradiation for different bias $I_{C}$, given by (3)

$$
\Delta\left(\frac{1}{h_{f e}}\right)=\left|\frac{1}{h_{f e l}}-\frac{1}{h_{f e o}}\right|
$$

where $h_{f e o}$ is the reading of $h_{f e}$ before the irradiation exposure and $h_{f e l}$ is the reading of $h_{f e}$ after being irradiated.

The decrement of $I_{C}$ and also increment of $I_{B}$ while exposed to gamma radiation leads to the reduction of $h_{f e}$. This eventually cause the $\left(\frac{1}{h_{f e}}\right)$ to increase as clearly evidenced in the plot shown in Fig. 4.

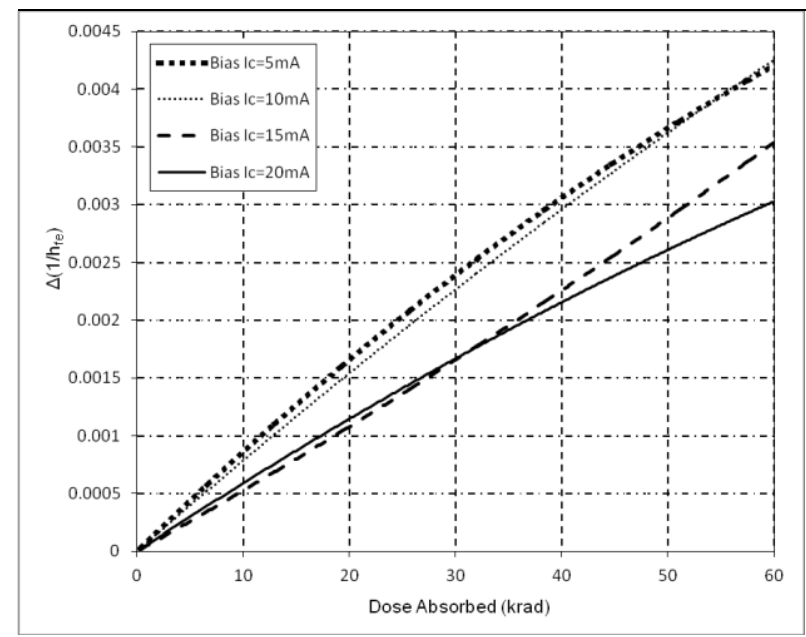

Figure 4: $\Delta\left(\frac{1}{h_{f e}}\right)$ of the BJT 2N3904 during irradiation at different bias $I_{C}$ for ON mode radiation.

The degradation in these performance parameters is ascribed to the effects of photon interaction with the silicon lattice. At post-irradiation, BJT establish minor annealing effect and shows slight increment in $I_{C}$ and decrement in $I_{B}$. Hence, this causes an improvement in the $h_{f e}$ as shown in Fig. 5. 


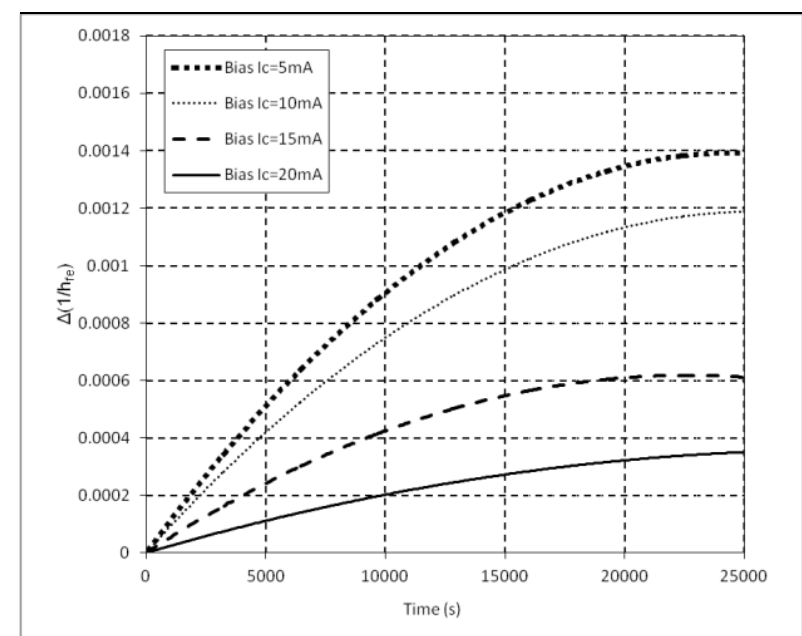

Figure 5: $4\left(\frac{1}{h_{f e}}\right)$ of the BJT 2N3904 at post-irradiation at different bias $I_{C}$ for ON mode radiation.

The $4\left(\frac{1}{h_{f e}}\right)$ of the BJT 2N3904 after exposure to a total dose of $60 \mathrm{krad}$ at OFF mode is graphically

depicted at Fig. 6. For the relative change of $h_{f e}$ at post-irradiation for different bias $I_{C}$, it is observed that the percentage of increase is higher at lower bias operating $I_{C}$. This means that the recovery in the parameter of $I_{C}$ and $I_{B}$ is lower during post-irradiation for higher bias $I_{C}$.

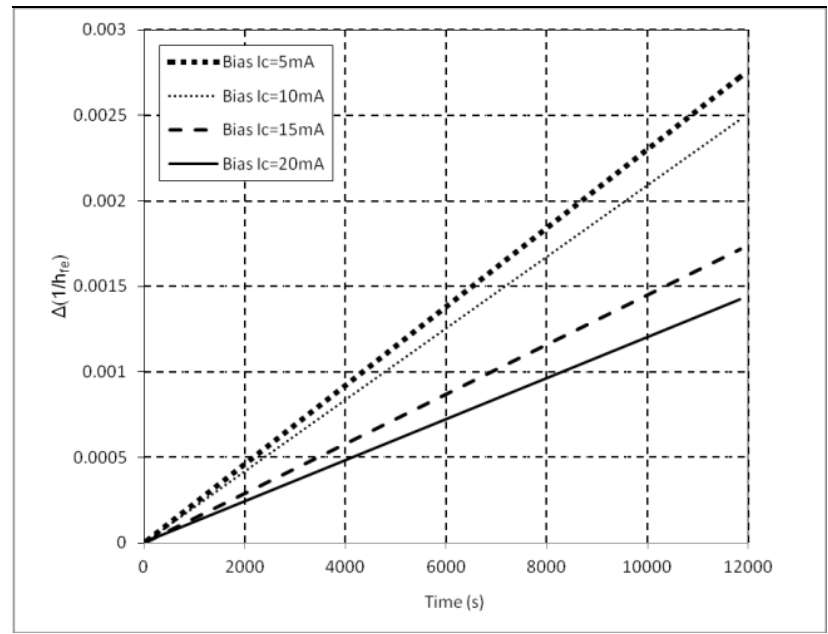

Figure 6: $\Delta\left(\frac{1}{h_{f e}}\right)$ of the BJT 2N3904 at post-irradiation at different bias $I_{C}$ for OFF mode radiation.

\section{Radiation Effect In The Surface Recombination Velocity Of BJT}

In thermal equilibrium, the generation and recombination rates in the BJTs are equal. The external excitation due to photons by gamma-rays was found to cause a decrease in the recombination factor of the BJT. This is as the deposition energy of the gamma-rays cause the generation of electrons and holes, inducing a nonequilibrium condition which increases the recombination rate in order to achieve the equilibrium condition. Hence, this increasing the surface recombination velocity, $S\left(D_{\gamma}\right)$ and therefore, decreasing the recombination factor in the BJT.

The recombination factor of BJT can be expressed as (4)

$$
\delta=\frac{J_{n E}+J_{p E}}{J_{n E}+J_{R}+J_{p E}} \approx \frac{J_{n E}}{J_{n E}+J_{R}}=\frac{1}{1+J_{R} / J_{n E}}
$$


$J_{n E}$ is the current densities due to the diffusion of minority carrier electrons in the base and can be expressed as (5)

$$
J_{n E}=J_{s 0} \exp \left(\frac{e V_{B E}}{k T}\right)
$$

$J_{R}$ is the current densities due to the recombination of carriers in the forward biased EBJ and can be expressed as (6)

$$
J_{R}=\frac{e x_{B E} n_{i}}{2 \tau_{0}} \exp \left(\frac{e V_{B E}}{2 k T}\right)=J_{r 0} \exp \left(\frac{e V_{B E}}{2 k T}\right)
$$

Where $\quad J_{s 0}=$ zero bias saturation current density

$J_{r 0}=$ zero bias recombination current density

The $\delta$ can then be expressed as (7)

$$
\delta=\frac{1}{1+\frac{J_{r 0}}{J_{s 0}} \exp \left(\frac{-e V_{B E}}{2 k T}\right)}
$$

The value of $J_{s 0}$ and $J_{r 0}$ which are applied for this modeling are $10^{-11} \mathrm{~A} / \mathrm{cm}^{2}$ and $10^{-8} \mathrm{~A} / \mathrm{cm}^{2}$ respectively.

From Fig. 7, it is observed that the recombination factor approaches unity when $V_{B E}$ increases as the recombination current becomes less dominant. Besides, the exposures of gamma rays deplete the recombination factor of BJT. The OFF mode radiation shows a greater depletion compared to the ON mode radiation.

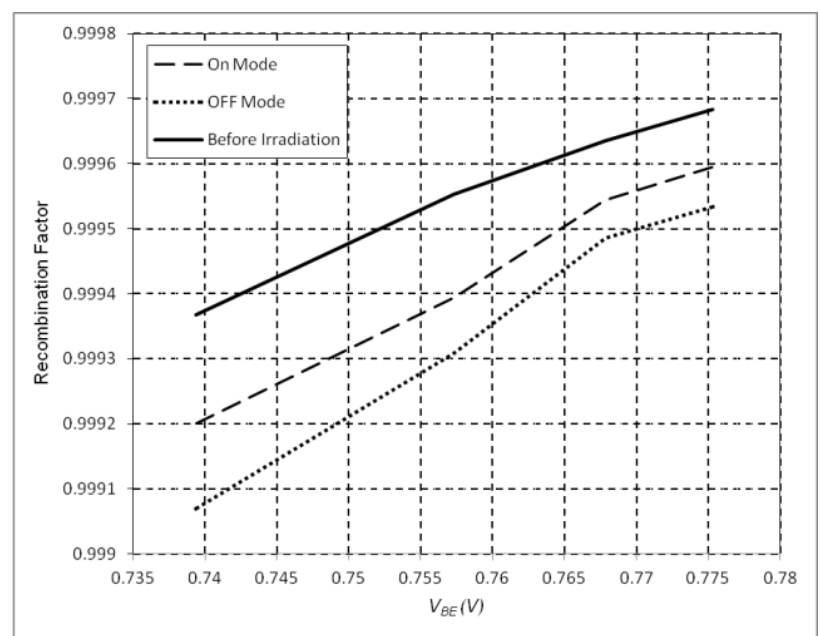

Figure 7: Recombination factor $(\delta$ ) of BJT 2N3904 at before irradiation and after exposed to a total dose radiation of $60 \mathrm{krad}$ gamma rays at $\mathrm{ON}$ and $\mathrm{OFF}$ mode.

\section{Radiation Effect In The Transconductance Of BJT}

Transconductance, $g_{m}$ which is defined as in (8) is found to be depleted significantly after being exposed to a total dose of $60 \mathrm{krad}$ gamma-rays.

$$
g_{m}=\frac{\partial I_{C}}{\partial V_{B E}}=\frac{e I_{C}}{k T}=\frac{e h_{f e} I_{B}}{k T}
$$

From the result as shown in Fig. 8, it is observed that performance of the BJT which is radiated at ON mode is more resistant to gamma-rays when being compared with that irradiated at OFF mode. 


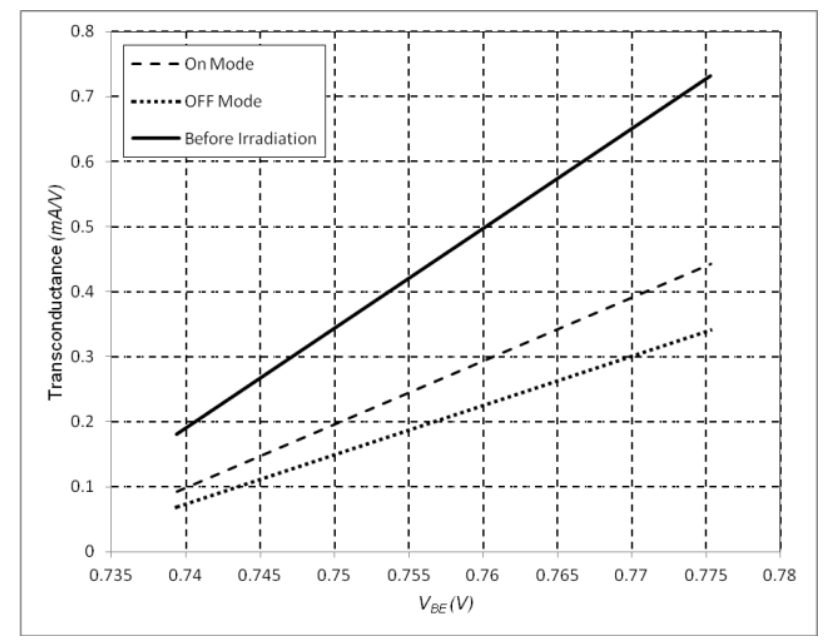

Figure 8: Transconductance $\left(g_{m}\right)$ of the BJT 2N3904 at before irradiation and after exposure to a total dose radiation of $60 \mathrm{krad}$ gamma rays at $\mathrm{ON}$ and $\mathrm{OFF}$ mode.

\section{Comparison T-Test For The Radiation Damage Of ON And OFF Mode Radiation}

The t-test is applied when there is a necessity to assess the means of two group results at two variables is statistically different from each other. Thus, t-test is carried out in this chapter to assess the significance of the difference between the means of $h_{f e}$ at two different radiation modes. The BJTs' $h_{f e}$ after being exposed to a total dose of $60 \mathrm{krad}$ at ON and OFF mode are tabulated in Table 1.

Table 1: Data statistics for average $h_{f e}$ of the BJT 2N3904 at bias $I_{C}=5 \mathrm{~mA}$ after exposure to a total dose radiation of

$60 \mathrm{krad}$ gamma rays at ON and OFF mode

\begin{tabular}{|c|c|}
\hline ON mode $h_{f e}$ & OFF mode $h_{f e}$ \\
\hline 113.9953 & 83.48111 \\
\hline 114.1784 & 84.05446 \\
\hline 114.3232 & 86.21775 \\
\hline 114.4143 & 86.28247 \\
\hline 114.5335 & 86.34581 \\
\hline 114.5968 & 86.51698 \\
\hline 114.6402 & 86.73426 \\
\hline 114.6943 & 86.83527 \\
\hline 114.7664 & 86.92604 \\
\hline 114.7954 & 86.99119 \\
\hline
\end{tabular}

Table 2: t-Test: Two- sample assuming unequal variances for data extracted from Table 1.

\begin{tabular}{|c|c|c|}
\hline & ON mode & OFF mode \\
\hline Mean & 114.4938 & 86.03853 \\
\hline Variance & 0.069164 & 1.522883 \\
\hline Observations & 10 & 10 \\
\hline Hypothesized Mean Difference & 0 & \\
\hline df & 10 & \\
\hline t Stat & 71.31556 & \\
\hline $\mathrm{P}(\mathrm{T}<=\mathrm{t})$ one-tail & $3.58 \mathrm{E}-15$ & \\
\hline $\mathrm{t}$ Critical one-tail & 1.812461 & \\
\hline $\mathrm{P}(\mathrm{T}<=\mathrm{t})$ two-tail & $7.17 \mathrm{E}-15$ & \\
\hline $\mathrm{t}$ Critical two-tail & 2.228139 & \\
\hline
\end{tabular}

Null hypothesis $\left(H_{0}\right)$ : There is no significant difference between the $h_{f e}$ of ON mode and OFF mode radiation after exposure to a Total Dose Radiation of $60 \mathrm{krad}$ gamma rays.

Alternative hypothesis: There is a significant difference between the $h_{f e}$ of ON mode and OFF mode radiation after exposure to a Total Dose Radiation of 60 krad gamma rays. 
Critical P level $(\alpha$ level $)=\mathbf{0 . 0 5}$

Option $1: \mathrm{P}(\mathrm{T}<=\mathrm{t})$ two-tail

$H_{0}$ is rejected if "P $(\mathrm{T}<=$ t) two-tail" $<$ critical $\mathrm{P}$ level (commonly 0.05$)$

$\mathrm{P}(\mathrm{T}<=\mathrm{t})$ two-tail $=\mathbf{7 . 1 7 E}-15$ which is $<0.05$ so $H_{0}$ is rejected

Option 2: t Stat

$H_{0}$ is rejected if t Stat is not in range of - (t Critical two-tail) and + (t Critical two-tail)

$\mathrm{t}$ Stat $=$ 71.316. It is out of range between $\mathrm{t}$ critical two-tail $(-2.228$ up to 2.228$) . H_{0}$ is rejected.

These results show that there is significant difference between means of $h_{f e}$ at ON mode and OFF mode radiation after exposure to a total dose radiation of $60 \mathrm{krad}$ gamma rays.

From the results of t-test conducted, it draws a conclusion that the operating mode of the BJT when irradiated by gamma-rays contributes a major variation in the degree of damage. This shows that the trapped charge, electric field and bulk damage induced by $\boldsymbol{\gamma}$-rays have a bigger impact for the devices operating in OFF mode. The absent of self-annealing effect in the BJT during irradiation is also one of the crucial effects which leads to the higher degradation in the OFF mode system.

\section{Research Discussions}

The operation of the BJT is based on the charge-carrier diffusion. In an NPN BJT, electrons emitted by the n-type emitter layer will diffuse through the middle material (base) and then collected at the collector region. If the NPN BJT were at perfect condition, all the emitted electrons will be collected while some might be lost through recombination with holes in the base. Therefore, the parameter $h_{f e}$, which is defined as the ratio of current that reaches the collector to the amount that recombines with the base, is a vital parameter of the BJT.

The most significant class of damage induced by gamma-rays in the BJT is the ionizing radiation effect. The second class of damage induced is the displacement damage and it is proportional to the nonionizing energy loss. Gamma-rays, having no rest mass, produce very little displacement damage and are very less damaging. The degradation is normally resulting from the interaction of a secondary electron produced by Compton scattering of the gamma-rays.

Under normal operating conditions, the valence band in the semiconductor is occupied by electrons at all energy levels while only very few electrons are available in the conduction band. The forbidden energy gap between the valence and conduction bands for silicon based BJT is $1.1 \mathrm{eV}$ at room temperature. The passage of the gamma radiation through the BJT carries more substantial excitation energy than the thermal agitation, thus, allowing more valence electrons to be excited into the conduction band.

The high energy of the radiation leads to the production of a large number of excited atoms along its track and causes the creation of the vacancies (holes) in the valence band. This phenomenon as illustrated in Fig. 9 is known as the creation of electron-hole pairs in the BJT device. The electrons in the conduction band are free to drift through the silicon material. These electrons are quite mobile and move to the most positive electrode while holes, with a rather complex transport mechanism, promote the probability of electrons trapping.

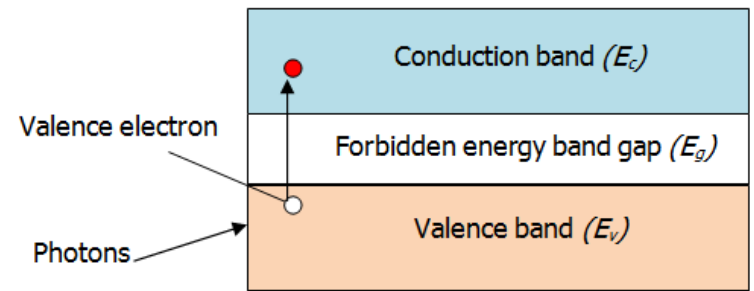

Figure 9: Excitation of electrons from valence to the conduction band.

In a state of normal operating environment, the electrons are pushed from the emitter into the base region when a relatively small $V_{B E}$ is applied. This creates a current flow across the emitter-base boundary. The electrons that get into the base region will then move swiftly towards the collector region. However, in this process flow, some of the free electrons that crossing the base might encounter a hole and recombination occur. Therefore, the increase of holes in the base region due to trapping as a result of irradiation will increase the probability of recombination and reducing the number of electrons that reaches the collector region. This can be seen from the increase of non-ideal $I_{B}$ and decrease of $I_{C}$ which in turn leads to the reduction of $h_{f e}$. This research outcome hypothesis is as illustrated in Fig. 10 and Fig. 11. 


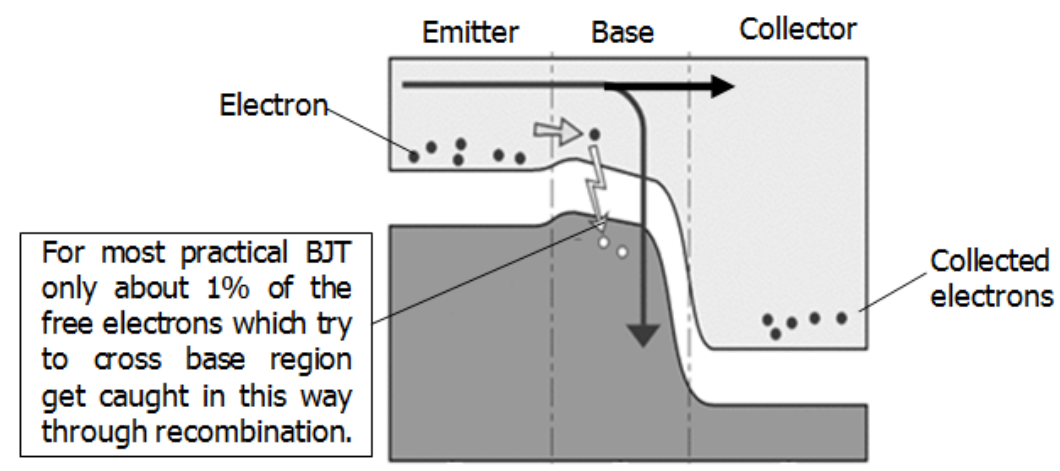

Figure 10: Normal charge flow in the BJT.

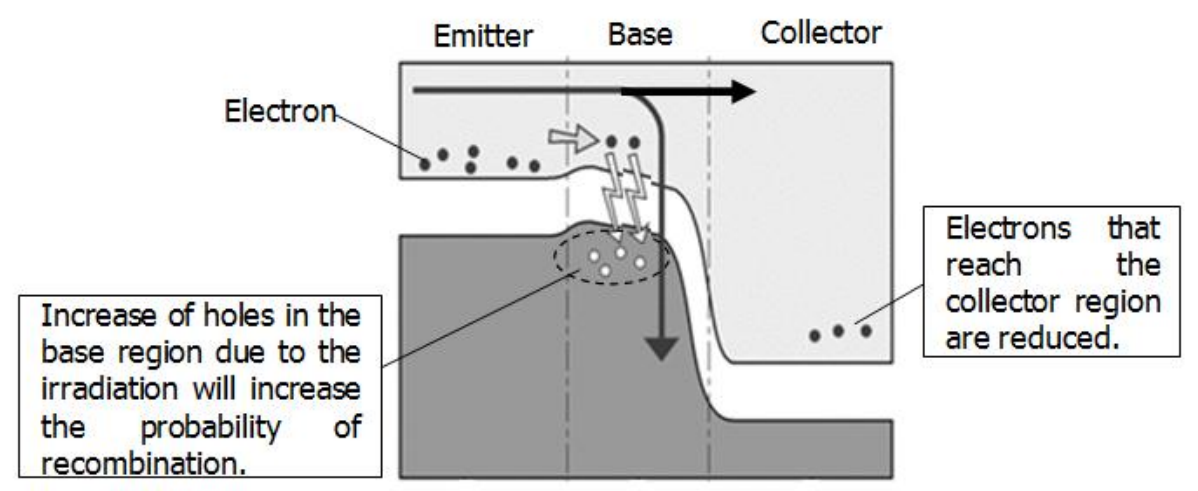

Figure 11: Charge flow in BJT due the irradiation.

\section{Acknowledgements}

The high energy of the gamma radiation allows more valence electrons to be excited to the conduction band. This leads to the production of a large number of excited atoms and increases the holes in the valence band. This phenomenon is known as the creation of electron-hole pairs in the BJT device. The increase of holes in the base region due to trapping will increase the probability of recombination and reducing the number of electrons that reaches the collector region.

In addition, the operating parameters and operating mode of the BJT can contribute to a difference in the degree of damage. Gamma radiation has a higher damaging effect on the OFF mode operating BJTs and for the BJT operating at a higher bias current $\left(I_{C}=20 \mathrm{~mA}\right)$, the degradation formed at both the $I_{C}$ and $I_{B}$ are found to be minimized significantly. This means that operating the irradiated device at large current during the exposure can remove part of the damage. This defect annealing maybe related to self-heating or more appropriately known as athermal diffusion mechanism.

\section{Acknowledgements}

The authors are thankful to Fundamental Research Grant Scheme (FRGS) 2013, Project No.: FRG0318-SG-1/2013, with title "Evaluation on Diffusion of Charge-Carrier in Semiconductor and nanostructure Devices and its dependency on Nuclear Radiation".

\section{References}

[1] P. J. Baines, and A. Côté, Promising Confidence- and Security-Building Measures for Space Security, A Safer Space Environment, 4, 2009, 5-16.

[2] F. P. Chee, H. F. A. Amir, and S. Salleh, Range distribution and electronic stopping power for Cobalt (Co) ions in Gallium Arsenide (GaAs) optoelectronic devices, Modeling, Simulation and Applied Optimization (ICMSAO), 2011 4th International Conference on. IEEE, 2011, 1-5.

[3] R. Krause-Rehberg and H. S. Leipner, Positron annihilation in semiconductors: defect studies, Vol. 127 (Springer, 1999).

[4] J. R. Srour, C. J. Marshall and P. W. Marshall, Review of displacement damage effects in silicon devices, Nuclear Science, IEEE Transactions on, 50(3), 2003, 653-670.

[5] P. J. Sellin and J. Vaitkus, New Materials for Radiation Hard Semiconductor Detectors, Nuclear Instruments and Methods in Physics Research Section A: Accelerators, Spectrometers, Detectors and Associated Equipment, 557(2), 2006, 479-489.

[6] X. Li, K. Shen, C. M. Huang, and L. Chu, A memory soft error measurement on production systems, 2007 USENIX Annual Technical Conference Proceedings. Santa Clara, CA, 2007, 275-280.

[7] T. Patel, New Fault Injection Approach for Network on Chip. IOSR Journal of Electronics and Communication Engineering, 5(2), 2013, 1-6. 
[8] T. Z. Fullem, Radiation detection using single event upsets in memory chips (ProQuest, 2006).

[9] American Society for Testing and Materials (ASTM), Electronics: Declarable Substances in Materials, vol. 10.04-2008 (Annual Book of ASTM Standards, Electrical Insulation and Electronics, ASTM International, 2008).

[10] A. L. Bogorad, J. J. Likar, S. K. Moyer, A. J. Ditzler, G. P. Doorley and R. Herschitz, Total Ionizing Dose and Dose Rate Effects in Candidate Spacecraft Electronic Devices, Radiation Effects Data Workshop, IEEE, 2008, 124-130.

[11] S. A. Francis, A. Dasgupta, and D. M. Fleetwood, Effects of Total Dose Irradiation on the Gate-Voltage Dependence of the 1/f Noise of nMOS and pMOS Transistors, Electron Devices, IEEE Transaction, 57(2), 2010, 503-510.

[12] N. A. Al- Hamdani, R. D. Al-Alawy, S. J. Hassan, Effect of gamma irradiation in the structural and optical properties of $\mathrm{ZnO}$ thin films, IOSR Journal of Computer Engineering, 16(1), 2014, 11-16.

[13] Department of Defense, MIL-STD-883 Test Method 1019.7 Ionizing radiation (Total Dose) test procedure (Test Method Standard Microcircuits, 2006)

[14] Poivey, C, Radiation Hardness Assurance for Space System, (NASA GSFC, 2009). 